\title{
Avaliação Pró-ativa da Deterioração de Sistemas de Informação por meio de Medidas de Gestão
}

\author{
André Luiz Pimentel Queiroz ${ }^{1}$ \\ Nicolas Anquetil ${ }^{1}$ \\ Káthia Marçal de Oliveira ${ }^{1}$
}

\begin{abstract}
Resumo: Questões relacionadas à modernização de sistemas de informação têm recebido maior atenção e relevância. Muitos métodos têm sido propostos para apoiar uma organização na escolha da melhor alternativa para modernização de um sistema. Entretanto, esses métodos não fornecem informações necessárias para a identificação precoce da deterioração das aplicações, sendo utilizados quando já se decidiu por realizar alguma ação para modernização da aplicação, como uma forma reativa de lidar com o problema. Este artigo apresenta uma abordagem baseada em medição com o objetivo de monitorar continuamente a deterioração de um sistema, permitindo ao gestor realizar ações de ajuste o mais cedo possível. Aplicações práticas em diferentes sistemas de uma grande empresa mostram a viabilidade de coleta das medidas e indicam os sistemas em deterioração.
\end{abstract}

Palavras-chave: Deterioração, Medidas, Sistemas Legados, Modernização de Sistemas.

1 Universidade Católica de Brasília - SGAN 916 Norte Av. W5- 70.790-160 Brasília- DF - Brasil andreluizqueiroz@gmail.com, \{nicolas,kathia\}@ucb.br 


\begin{abstract}
Issues related to the decision on software modernization have received more attention and importance. Many methods have been proposed to support an organization's choice of the best alternative for a system modernization. However, these methods do not supply the necessary information to the early identification of system deterioration, being used when it is already decided for some modernization action in the system application, as a reactive way of deal with the problem. This article presents an approach based on measurement that aims to continuously monitor the system deterioration, allowing managers to perform adjustment actions as soon as possible, aiming to extend its useful life. Practical applications in different systems of a large company show the viability of collecting these measures and indicate the systems in deterioration.
\end{abstract}

Keywords: Deterioration, Measures, Legacy Systems, Software modernization. 


\section{Introdução}

As decisões a respeito da continuidade dos investimentos na manutenção de um sistema, ou na sua substituição, têm crescido em importância, pois além de envolver valores financeiros expressivos, podem causar mudanças significativas na vida dos seus usuários e até mesmo, no sucesso de uma organização [1]. Nesse contexto, vários métodos de apoio à decisão sobre a modernização de sistemas têm sido propostos [2], [3], [4], [5], [6]. As estratégias de modernização propostas nestes métodos, como reengenharia, substituição ou re-implementação, são normalmente utilizadas quando já se possui a percepção de que alguma ação deve ser executada para modernização de um sistema, sendo, portanto, uma forma reativa de lidar com o problema [7].

A importância da pró-atividade nos métodos de suporte à decisão sobre a modernização de sistemas destaca-se diante dos atuais níveis de utilização e relevância destes ativos nas organizações. Esta pró-atividade concretiza-se através da detecção precoce da deterioração dos sistemas de informação, da otimização do uso destes recursos e da possibilidade do tratamento de problemas em seus estágios iniciais.

Segundo Harris e McRory [8], mudanças contínuas nos sistemas podem levar à degradação da qualidade técnica e do valor do sistema para a organização. Para detectar possíveis desvios, devem ser executadas avaliações periódicas do desempenho técnico, operacional e funcional dos sistemas e, a partir destes resultados, elaborado um plano de ação corretivo.

Este contexto remete ao propósito desse trabalho que é beneficiar-se dos métodos existentes de apoio à decisão e de normas de qualidade de produto [9], com o objetivo de desenvolver uma abordagem para a monitoração contínua, com medidas de gestão úteis à identificação precoce da deterioração de sistemas de informação, de forma a permitir que gestores possam realizar ações preventivas com a possibilidade de tratamento de problemas em seus estágios iniciais, antes da adoção das estratégias de modernização propostas nos métodos tradicionais.

Nas próximas seções deste artigo serão apresentadas: uma descrição sucinta de métodos de suporte a decisão sobre a modernização de sistemas (Seção 2), os conceitos considerados na definição da deterioração de sistemas (Seção 3) e a abordagem para avaliação de sistemas proposta neste trabalho (Seção 4). Finalizando, na Seção 5 serão discutidos os resultados obtidos na prática e na Seção 6, as conclusões deste trabalho.

\section{Métodos de Suporte a Decisão sobre Modernização de Sistemas}

De maneira geral, os métodos de suporte à decisão sobre modernização de sistemas se baseiam na definição de critérios que, uma vez avaliados, permitam identificar qual a alternativa mais adequada de modernização para o sistema [2], [3], [4], [5], [6], [8]. 
Sneed [2], um dos autores de maior destaque no estudo sobre estes métodos, propõe um conjunto de passos que incluem mensurar a qualidade técnica e o valor de negócio, e que tem por objetivo avaliar se o método de reengenharia é a melhor alternativa para a modernização de aplicações. Aversano et al [6] também utilizam critérios para avaliar a qualidade técnica e o valor para o negócio dos componentes das aplicações, e a partir dos critérios considerados não satisfatórios, definem a estratégia de evolução do sistema. De acordo com estes modelos, cada estratégia de modernização de sistemas legados está relacionada com a melhoria da qualidade de um ou mais critérios de avaliação.

Uma outra metodologia é proposta por Bennet, Ramage e Munro [10]. Neste trabalho é apresentado um modelo denominado SABA (Software As a Business Asset), baseado na elaboração de cenários e composto por duas fases. A primeira fase consiste em explorar os objetivos da organização construindo diferentes cenários para o seu futuro. No segundo passo, visando apoiar os cenários de negócio elaborados, são construídos cenários tecnológicos a partir das estratégias de evolução dos sistemas legados.

Mais recentemente, Harris e McRory [8] propuseram um conjunto de indicadores que representassem o desempenho técnico, operacional e funcional dos sistemas. De acordo com este método, para detectar possíveis desvios de desempenho, uma avaliação subjetiva deve ser realizada para cada um destes indicadores, resultando em uma nota, ou conceito geral, para o sistema. Ações corretivas poderiam ser seguidas de acordo com as notas finais.

De forma geral, os métodos existentes trabalham com a premissa que o sistema avaliado já apresenta algum desvio na sua qualidade e demonstram, também, pouca preocupação em discutir como os critérios utilizados para a decisão podem ser mensurados, exceção feita as propostas de Sneed [2] e Visaggio [4]. Além disso, mesmo o método proposto por Harris e Mcroy [8] que foi o único a apresentar características de pró-atividade, propõe uma avaliação subjetiva dos indicadores e um conjunto genérico de ações corretivas para os sistemas.

\section{Deterioração de Sistemas}

Para Lehman et al [11], a qualidade de um sistema ou software em constante evolução será comprometida a menos que este sistema seja rigorosamente mantido e adaptado para as mudanças operacionais em seu ambiente. Este enunciado é conhecido como a sétima lei de Lehman, a lei do Declínio da Qualidade (Declining Quality).

De acordo com Jermakovics, Scotto e Succi [12], o declínio da qualidade de um sistema representa somente parte das conseqüências da sua evolução: "o problema real surge quando se torna cada vez mais difícil atender a requisitos de modificações nos sistemas". O aumento na dificuldade em modificar um sistema é a idéia principal em que se baseiam alguns autores para definir conceitos semelhantes. Jermakovics, Scotto e Succi [12] definem que a deterioração de software (software deterioration) ocorre quando um sistema torna-se 
cada vez pior em sua estrutura, levando a um declínio de sua qualidade e no aumento do tempo para satisfazer a novos requisitos de negócio.

Gurp e Bosch [13] concluem como inevitável a ocorrência da erosão do design (desing erosion) de sistemas submetidos à pressão de modificações ao longo do tempo. Para Parnas [14] o envelhecimento do sistema (software aging) pode ser causado por dois motivos: pela falha em modificar um sistema visando atender a novos requisitos de negócio e como resultado de mudanças contínuas. Eick et al [15], concluem que a decadência do código (code decay) contribui para que a manutenção evolutiva de um sistema torne-se cada vez mais difícil e necessite de mais recursos.

Para Cimitile, Fasolino e Lanubile [16], a deterioração de software expressa a idade de um sistema como resultado das mudanças contínuas às quais este foi submetido e apresenta por sintomas típicos: (i) degradação do desempenho como resultado do aumento do tamanho e comprometimento de sua estrutura; (ii) degradação da confiabilidade, pelo número de novos erros inseridos por manutenções passadas; e (iii) degradação da capacidade de modificação, como resultado do fato de que um código degradado não é bem entendido nem bem documentado.

Entretanto, para Kajko-Mattsson [17] a degradação do desempenho está associada à operação contínua de um sistema e é identificada como o envelhecimento do processo de operação deste software (software process execution ageing). A degradação do código e da documentação de um sistema em evolução é que está relacionada com o envelhecimento do software como produto (software product ageing).

Em resumo, pode-se dizer que a deterioração de sistemas de informação se materializa no declínio da capacidade em manter e evoluir um sistema ao longo do seu ciclo de vida, no conseqüente aumento dos custos de sua operação e na dificuldade em satisfazer de maneira consistente às expectativas e novos requisitos das áreas de negócio.

\section{Uma Abordagem para Avaliação da Deterioração}

Na visão de Basili, Caldiera e Rombach [18], para que uma organização possa medir de maneira eficiente, ela deve primeiro especificar os objetivos para si e seus projetos, associar estes objetivos a dados que possam defini-los operacionalmente, e por fim, prover um framework para auxiliar na interpretação destes dados com relação aos objetivos definidos. Este é o princípio no qual se baseia a abordagem GQM - Goal Question Metric.

Utilizando o GQM e de acordo com o formato proposto por [19], o objetivo deste trabalho pode ser então formalizado como:

Analisar um sistema de informação (software),

Com o propósito de monitorar,

Com respeito à sua deterioração,

Sob ponto de vista de gestores da aplicação, 
No contexto de uma organização proprietária do sistema, responsável por definir requisitos para sua manutenção evolutiva.

Uma vez definido o objetivo, o próximo passo da fase de definição do GQM diz respeito à identificação de questões que caracterizem o objeto da avaliação, de acordo com o ponto de qualidade avaliado e com respeito ao ponto de vista selecionado [18]. Especificamente neste estudo, as questões devem caracterizar a monitoração sobre a deterioração de um sistema de informação em operação, do ponto de vista de seus gestores.

Como visto nas definições da seção anterior, as características gerais da deterioração de um sistema estão baseadas no declínio da capacidade em manter e evoluir um sistema ao longo do seu ciclo de vida, no conseqüente aumento dos custos de sua operação e na dificuldade em satisfazer de maneira consistente às expectativas e novos requisitos das áreas de negócio. Dessa forma, para avaliar a deterioração têm-se as seguintes questões:

Q1- A manutenibilidade do sistema está sendo comprometida?

Q2 - Os custos do sistema são elementos ofensores à continuidade de sua operação?

Q3 - Qual o nível de satisfação dos usuários com relação à utilização do sistema?

Para definir as medidas que visam atender a estes questões, decidiu-se investigar os critérios de avaliação de sistemas definidos nos métodos existentes de apoio à decisão sobre a modernização de sistemas e as medidas de manutenibilidade definidas na ISO/IEC 9126 [9].

Um total de 169 medidas foram identificadas, sendo 44 consideradas duplicadas em relação à sua definição ou forma de cálculo, resultando em um subconjunto de 125 medidas.

As 125 medidas foram, então, analisadas considerando seis premissas. As quatro primeiras premissas foram elaboradas considerando os itens definidos no objetivo do GQM: (i) medidas que tenham foco na deterioração de sistemas (visto que elas foram definidas considerando outro contexto), (ii) que representem o contexto de organizações usuárias de sistemas, (iii) e a visão de gestores de sistemas. No que se refere ao propósito de monitorar, foram consideradas as definições de Kitchenham e Walker [20] de que existem dois tipos básicos de processos de monitoração de medidas: monitoração por etapas (checkpoint monitoring) caracterizada pela avaliação das medidas após a conclusão de alguma ação ou etapa e a monitoração baseada no tempo (time-based monitoring) onde as medidas são avaliadas ao decorrer do tempo. Como o objetivo da abordagem proposta é monitorar a deterioração de um sistema de informação em operação, será utilizada a monitoração baseada no tempo, sendo consideradas, portanto, somente medidas que possam ser avaliadas ao longo de um período contínuo de operação do sistema. Dessa forma, estabeleceu-se como premissa (iv) a necessidade de evolução histórica.

A quinta (v) premissa considerada está relacionada com a maneira como as decisões a respeito dos sistemas de informação são elaboradas. Com o objetivo de tornar o processo de monitoração baseado em evidências relacionadas diretamente com a operação do sistema, foram excluídas da seleção as medidas que tivessem sua forma de apuração baseada na 
avaliação subjetiva. Além disso, como o objetivo é ter uma monitoração contínua do sistema, realizando avaliações periódicas ao longo do ano, a avaliação subjetiva poderia envolver custos altos sem o benefĩcio adequado, pois os avaliadores estariam repetindo constantemente as mesmas avaliações.

A última premissa considerada na abordagem é (vi) a utilização de medidas simples e factíveis de apuração com relação ao custo e frequiência de avaliação. Na visão de Koskinen et al [7], uma das principais críticas aos métodos de apoio à decisão é que estes assumem a disponibilidade de uma quantidade irreal de dados de entrada. Para Fenton e Neil [21] este é um dos principais problemas apontados sobre o distanciamento entre as medidas propostas nos estudos acadêmicos e a realidade praticada nas organizações. Para estes autores, a indústria tem adotado medidas simples e, rejeitado medidas complexas, pois, além da complexidade de apuração, seus resultados não se mostraram mais válidos que os resultados das medidas mais simples. Com isso, o propósito da abordagem é utilizar um conjunto de medidas que sejam simples de apurar, considerando seu esforço e custo, e ao mesmo tempo, úteis aos gestores das aplicações. A Tabela 1 apresenta alguns exemplos da análise das medidas identificadas considerando as seis premissas.

Analisando as 125 medidas, a grande maioria (96) foi descartada por não ter como foco a deterioração e somente 8 atenderam todos os critérios definidos: volume de falhas, tamanho de backlog, tamanho do esforço por demanda, produtividade, eficiência do ciclo de correções, custo de manutenção, tempo médio entre falhas e tempo de resposta do sistema. Para complementar a identificação de medidas dois procedimentos foram seguidos.

Inicialmente, baseado na proposta de Soligen e Berghout [19] com o uso de abstract sheets, foi analisado se existiam fatores que poderiam influenciar o resultado dessas medidas. Esses fatores seriam considerados como novas medidas e, portanto, também deveriam ser analisados considerando as seis premissas anteriormente definidas. A partir dessa análise, duas novas medidas foram acrescentadas: número de novas demandas, que impacta nas avaliações referentes ao backlog e produtividade, e, nível de utilização de hardware, que pode influenciar no tempo de resposta do sistema. Essas medidas são utilizadas apenas de forma complementar para a análise de medidas referente a ao backlog e tempo de resposta do sistema. O número de novas demandas, por exemplo, foi considerado suficiente para analisar algum impacto no backlog, embora outras medidas pudessem ser utilizadas (por exemplo, esforço para implementação). 
Tabela 1. Exemplos de análise das medidas

\begin{tabular}{|c|c|c|c|c|c|c|}
\hline Medida & $\begin{array}{c}\text { (i) } \\
\text { Foco } \\
\text { Deterioração } \\
\end{array}$ & $\begin{array}{c}\text { (ii) } \\
\text { Organização } \\
\text { Gestora }\end{array}$ & $\begin{array}{c}\text { (iii) } \\
\text { Visão } \\
\text { Gestor }\end{array}$ & $\begin{array}{l}\text { (iv) } \\
\text { Evolução } \\
\text { Histórica } \\
\end{array}$ & $\begin{array}{c}\text { (v) } \\
\text { Não } \\
\text { Subjetividade }\end{array}$ & $\begin{array}{c}\text { (vi) } \\
\text { Simplicidade }\end{array}$ \\
\hline $\begin{array}{l}\text { Aumento } \\
\text { da taxa de } \\
\text { erros [5] }\end{array}$ & $\begin{array}{l}\text { Sim - análise } \\
\text { histórica } \\
\text { pode indicar } \\
\text { a } \\
\text { deterioração } \\
\text { do sistema. }\end{array}$ & $\begin{array}{l}\text { Sim - } \\
\text { Contexto da } \\
\text { organização } \\
\text { gestora. }\end{array}$ & $\begin{array}{l}\text { Sim - } \\
\text { Informaçã } \\
\text { o relevante } \\
\text { para o } \\
\text { gestor do } \\
\text { sistema. }\end{array}$ & $\begin{array}{l}\text { Sim - } \\
\text { Passível de } \\
\text { avaliação } \\
\text { histórica. }\end{array}$ & $\begin{array}{l}\text { Sim - Pode ser } \\
\text { apurado } \\
\text { objetivamente. }\end{array}$ & $\begin{array}{l}\text { Sim - } \\
\text { Quantidade } \\
\text { de erros } \\
\text { apurados no } \\
\text { período. }\end{array}$ \\
\hline $\begin{array}{l}\text { Tamanho } \\
\text { do } \\
\text { Esforço } \\
\text { para } \\
\text { manutenç } \\
\text { ão[2] }\end{array}$ & $\begin{array}{l}\text { Sim - análise } \\
\text { histórica } \\
\text { pode indicar } \\
\text { a } \\
\text { deterioração } \\
\text { do sistema. }\end{array}$ & $\begin{array}{l}\text { Sim - } \\
\text { Contexto da } \\
\text { organização } \\
\text { gestora. }\end{array}$ & $\begin{array}{l}\text { Sim - } \\
\text { Informaçã } \\
\text { o relevante } \\
\text { para o } \\
\text { gestor do } \\
\text { sistema. }\end{array}$ & $\begin{array}{l}\text { Sim - } \\
\text { Passível de } \\
\text { avaliação } \\
\text { histórica. }\end{array}$ & $\begin{array}{l}\text { Sim - Pode ser } \\
\text { apurado } \\
\text { objetivamente. }\end{array}$ & $\begin{array}{l}\text { Sim - } \\
\text { Número de } \\
\text { horas por } \\
\text { manutenção. }\end{array}$ \\
\hline $\begin{array}{l}\text { Número } \\
\text { de } \\
\text { Variáveis } \\
\text { do } \\
\text { sistema } \\
{[7]}\end{array}$ & $\begin{array}{l}\text { Não - análise } \\
\text { histórica não } \\
\text { possui } \\
\text { relação com a } \\
\text { deterioração } \\
\text { do sistema. }\end{array}$ & $\begin{array}{l}\text { Não - } \\
\text { Relevante } \\
\text { para } \\
\text { organizações } \\
\text { desenvolvedo } \\
\text { ras de } \\
\text { sistemas. }\end{array}$ & $\begin{array}{l}\text { Não - } \\
\text { Informaçã } \\
\text { o de } \\
\text { caráter } \\
\text { técnico. }\end{array}$ & $\begin{array}{l}\text { Sim - } \\
\text { Passível de } \\
\text { avaliação } \\
\text { histórica. }\end{array}$ & $\begin{array}{l}\text { Sim - Pode ser } \\
\text { apurado } \\
\text { objetivamente. }\end{array}$ & $\begin{array}{l}\text { Sim - } \\
\text { Utilização de } \\
\text { ferramentas } \\
\text { de análise de } \\
\text { código. }\end{array}$ \\
\hline $\begin{array}{l}\text { Aumento } \\
\text { do tempo } \\
\text { de } \\
\text { resposta } \\
{[5]}\end{array}$ & $\begin{array}{l}\text { Sim - análise } \\
\text { histórica } \\
\text { pode indicar } \\
\text { a } \\
\text { deterioração } \\
\text { do sistema. }\end{array}$ & $\begin{array}{l}\text { Sim - } \\
\text { Impacta } \\
\text { diretamente a } \\
\text { organização } \\
\text { gestora. }\end{array}$ & $\begin{array}{l}\text { Sim - } \\
\text { Informaçã } \\
\text { o relevante } \\
\text { para o } \\
\text { gestor do } \\
\text { sistema. }\end{array}$ & $\begin{array}{l}\text { Sim - } \\
\text { Passível de } \\
\text { avaliação } \\
\text { histórica. }\end{array}$ & $\begin{array}{l}\text { Sim - Pode ser } \\
\text { apurado } \\
\text { objetivamente. }\end{array}$ & $\begin{array}{l}\text { Sim - Tempo } \\
\text { de resposta } \\
\text { das principais } \\
\text { funcionalidad } \\
\text { es do } \\
\text { sistema. }\end{array}$ \\
\hline $\begin{array}{l}\text { Qualidade } \\
\text { da } \\
\text { document } \\
\text { ação [3] }\end{array}$ & $\begin{array}{l}\text { Sim - análise } \\
\text { histórica } \\
\text { pode indicar } \\
\text { a } \\
\text { deterioração } \\
\text { do sistema. }\end{array}$ & $\begin{array}{l}\text { Sim - } \\
\text { Impacta } \\
\text { diretamente a } \\
\text { organização } \\
\text { gestora. }\end{array}$ & $\begin{array}{l}\text { Sim - } \\
\text { Informaçã } \\
\text { o relevante } \\
\text { para o } \\
\text { gestor do } \\
\text { sistema. }\end{array}$ & $\begin{array}{l}\text { Sim - } \\
\text { Passível de } \\
\text { avaliação } \\
\text { histórica. }\end{array}$ & $\begin{array}{l}\text { Não - Requer } \\
\text { avaliação } \\
\text { subjetiva. }\end{array}$ & $\begin{array}{l}\text { Não - } \\
\text { Relação entre } \\
\text { a frequência } \\
\text { e custo da } \\
\text { apuração } \\
\text { subjetiva. }\end{array}$ \\
\hline $\begin{array}{l}\text { Impacto } \\
\text { das } \\
\text { Mudanças } \\
{[2]}\end{array}$ & $\begin{array}{l}\text { Sim - análise } \\
\text { histórica } \\
\text { pode indicar } \\
\text { a } \\
\text { deterioração } \\
\text { do sistema. }\end{array}$ & $\begin{array}{l}\text { Sim - } \\
\text { Impacta } \\
\text { diretamente a } \\
\text { organização } \\
\text { gestora. }\end{array}$ & $\begin{array}{l}\text { Não - } \\
\text { Informaçã } \\
\text { o de } \\
\text { caráter } \\
\text { técnico. }\end{array}$ & $\begin{array}{l}\text { Sim - } \\
\text { Passível de } \\
\text { avaliação } \\
\text { histórica. }\end{array}$ & $\begin{array}{l}\text { Sim - Pode ser } \\
\text { apurado } \\
\text { objetivamente. }\end{array}$ & $\begin{array}{l}\text { Sim - } \\
\text { Apuração } \\
\text { com } \\
\text { ferramentas } \\
\text { de análise de } \\
\text { código. }\end{array}$ \\
\hline
\end{tabular}


Uma segunda análise foi feita a partir da entrevista com diferentes gestores de sistemas para os quais foi solicitado responder a seguinte questão: "Que informações gostaria de ter disponíveis sobre um sistema que está em operação para avaliar se ele está em deterioração?". Essas entrevistas foram conduzidas individualmente, com 5 gestores com experiências entre 10 e 25 anos na indústria de sistemas de informação. Os gestores indicaram várias informações que consideravam importantes para avaliar uma deterioração. Analisando a consolidação dessas informações (Tabela 2), muitas já estavam sendo contempladas com as medidas já consideradas, algumas não atendiam as premissas definidas anteriormente e outras resultaram em duas novas medidas a serem avaliadas de forma a verificar o comprometimento do orçamento da empresa com a manutenção do sistema: custo médio por demanda, que tem por objetivo avaliar os valores gastos no atendimento de novos requisitos de negócio; e, o percentual de participação do orçamento, que visa identificar a participação dos custos de operação do sistema com relação aos custos totais com TI da organização.

Tabela 2. Informações citadas pelos gestores

Necessidades Citadas pelos Gestores

Quantidade de erros

Quantidade de manutenções evolutivas

Tempo de atendimento das melhorias

Tecnologias utilizadas pelos fornecedores

Quantidade de interfaces (integrações) ativas com outros sistemas

Quantidade de solicitações de novas interfaces (integrações) com outros sistemas

Quantidade de funcionalidades sem uso

Tempo de execução de processos críticos

Qualidade da documentação do sistema

Tamanho de backlog

\section{Análise}

Informação contemplada na medida Volume de Falhas

Informação contemplada na medida Produtividade

Informação contemplada na medida Produtividade

Não atende a premissa (iv) Evolução Histórica

Não atende a premissa (i) Foco

Deterioração

Não atende a premissa (i) Foco

Deterioração

Não atende a premissa (vi) Simplicidade

Informação contemplada na medida

Tempo de Resposta do Sistema

Não atende a premissa (v) Não

Subjetividade

Informação contemplada na medida

Tamanho do backlog 
Tabela 2 (cont.). Informações citadas pelos gestores

\section{Necessidades Citadas pelos Gestores}

Aumento da Complexidade para implementação das demandas

Histórico do custo de manutenção

\section{Análise}

Informação contemplada na medida Tamanho do Esforço por Demanda

Informação contemplada na evolução da medida Custo de Manutenção

\section{Comprometimento do orçamento da empresa Definição de novas medidas}

Quantidade de acessos ao sistema

Nível de Satisfação

Número de usuários ativos

Número de novas solicitações de acesso
Não atende a premissa (i) Foco

Deterioração

Não atende a premissa (v) Não

Subjetividade

Não atende a premissa (i) Foco

Deterioração

Não atende a premissa (i) Foco

Deterioração

Informação contemplada na medida Tempo de Resposta do Sistema

Desempenho do sistema compa
serviço acordado com o usuário

Dessa forma, na confecção do GQM final (Tabela 3) para questão Q1 (a manutenibilidade do sistema está sendo comprometida?) foram selecionadas medidas relacionadas com a ocorrência de falhas (M1 e M6) e com a implementação de novos requisitos de negócio (M2, M3, M4, M5). Na questão Q2 (Os custos do sistema são elementos ofensores à continuidade de sua operação?) estão sendo monitoradas as evoluções dos custos de manter o sistema em operação (M8 e M9) e do atendimento de novos requisitos (M7). O nível de satisfação dos usuários, questão Q3 (Qual o nível de satisfação dos usuários com relação à utilização do sistema?), está sendo monitorado através do volume e freqüência de falhas na operação do sistema (M1 e M10), da capacidade de atendimento às manutenções evolutivas (M2 e M4) e da operação e uso do sistema (M11 e M12).

A periodicidade de coleta para a maioria das medidas (todas com exceção de M8 e M9) foi definida como trimestral, de forma a permitir uma evolução histórica ao longo do ano (premissa (iv)) em intervalos de tempo que mostre possíveis variações. Para as medidas M8 e M9 foi definida coleta anual por serem medidas mais relacionadas a custos do sistema.

De acordo com o conjunto de medidas definido, alguns pré-requisitos para a sua apuração devem ser considerados. O primeiro deles é que a organização deve possuir o registro das falhas ocorridas na operação dos sistemas. Este controle será essencial para o cálculo das medidas M1, M6 e M10. Adicionalmente, o controle das demandas por manutenção evolutiva dos sistemas, também será necessário para a apuração das medidas 
M2, M3, M4, M5 e M7. As medidas M11 e M12 necessitam de monitoramento do ambiente produtivo do sistema através de ferramentas específicas que registram, armazenam e alarmam quando algum componente da sua infra-estrutura apresenta desempenho inferior ao desejado, por exemplo, como uso de agentes instalados nos servidores que monitoram desempenho das aplicações, percentual de memória livre da máquina, desempenho de entrada/saída, etc.. Fechando a lista de pré-requisitos, as organizações devem possuir a identificação dos custos associados à operação dos sistemas, conforme descritos no procedimento de coleta das medidas M8 e M9.

Finalmente, para permitir a análise da deterioração foi necessário definir critérios de decisão que permitam determinar a necessidade de uma ação ou de uma maior investigação [22]. Os critérios de decisão são representados pelos valores históricos das medidas ao longo da operação do sistema. Especificamente, para as medidas com a periodicidade de apuração anual, utilizou-se os valores apurados no ano anterior como critério de decisão para a identificação da deterioração. Assim, estas medidas são consideradas como em deterioração caso os valores apurados no ano atual sejam superiores, ou piores, que os valores históricos apurados.

Para as medidas com a periodicidade de apuração trimestral, médias aritméticas históricas foram utilizadas como forma de detectar um comportamento de deterioração. Assim, estes valores médios serviram como pontos de referência para a identificação da deterioração, sendo, portanto, as bases para os critérios de decisão. Para Pickard [23], a utilização de médias numéricas é uma maneira bastante usual de determinar um "ponto central" necessário na identificação de valores anormais.

Duas médias aritméticas foram utilizadas na análise do desempenho das medidas. A primeira é a média acumulada histórica, sendo calculada como somatório dos valores de todos os trimestres dividido pela quantidade total de trimestres apurados. A segunda média, com o objetivo de analisar o desempenho recente da medida, representa a média móvel dos últimos 4 (quatro) trimestres, sendo calculada como somatório dos valores destes trimestres, dividido por 4 (quatro).

A análise sobre a deterioração da medida no trimestre é realizada, portanto, da seguinte forma sobre o valor do trimestre:

- Alto sinal de deterioração - é maior ou igual, que a média móvel E maior ou igual, que a média acumulada, independente da relação entre as médias;

- Médio sinal de deterioração - é maior ou igual, que a média móvel OU maior ou igual, que a média acumulada, independente da relação entre as médias;

- Baixo sinal de deterioração - é menor que a média móvel E menor que a média acumulada E a média móvel é maior que a média acumulada; e

- Nenhum sinal de deterioração - é menor que a média móvel E menor que a média acumulada $\mathrm{E}$ a média móvel é menor que a média acumulada. 
Desta forma, a cada apuração trimestral, os gestores têm a sua disposição um conjunto de medidas que, de acordo com os critérios de decisão definidos, representam os sinais de deterioração das medidas definidas no estudo. $\mathrm{O}$ escopo desta abordagem não compreende a automação da decisão a respeito da deterioração do sistema e sim, prover, aos gestores, uma ferramenta de apoio à identificação destes sinais.

Os resultados das medidas e da análise são apresentados aos gestores como diferentes painéis de controle: um painel consolidado com os valores, com seu valor apurado no trimestre, suas médias histórica e móvel e o respectivo sinal de deterioração, e painéis detalhados para cada questão com gráficos apresentando os valores históricos. Exemplos desses painéis serão apresentados na próxima seção.

Tabela 3. Medidas para monitoração da deterioração de sistemas de informação

\begin{tabular}{|c|c|c|c|c|}
\hline Questão & Medida & Fórmula & Escala & Descrição \\
\hline $\begin{array}{l}\text { Q1 } \\
\text { Q3 }\end{array}$ & $\begin{array}{l}\text { M1 - } \\
\text { Volume } \\
\text { de } \\
\text { Falhas } \\
\text { (Adapta } \\
\text { da de } \\
{[5] \text { ) }}\end{array}$ & $\begin{array}{l} \\
\text { Quantidad } \\
\text { e falhas no } \\
\text { período }\end{array}$ & $\begin{array}{l}\text { Absoluta } \\
\text { - } \\
\text { Números } \\
\text { inteiros, } \\
\text { de zero } \\
\text { até } \\
\text { infinito. }\end{array}$ & $\begin{array}{l}\text { Quantidade de falhas do período registradas na } \\
\text { operação do sistema. } \\
\text { Propósito: } \\
\text { Verificar a evolução histórica do volume de } \\
\text { falhas da aplicação pode indicar a deterioração } \\
\text { da manutenibilidade, pois reflete a dificuldade } \\
\text { em manter um sistema em operação. } \\
\text { Procedimento de coleta: } \\
\text { 1. Contar o número de falhas, a partir dos } \\
\text { registros de ocorrência de falhas no sistema. }\end{array}$ \\
\hline $\begin{array}{l}\text { Q1 } \\
\text { Q3 }\end{array}$ & $\begin{array}{l}\text { M2 - } \\
\text { Tamanh } \\
\text { o de } \\
\text { Backlog } \\
\text { [24] }\end{array}$ & $\begin{array}{l}\text { (backlog } \\
\text { anterior) } \\
+\left(\sum\right. \\
\text { demanda } \\
\text { s abertas }) \\
-\left(\sum\right. \\
\text { demanda } \\
\text { S } \\
\text { satisfeita } \\
\text { s no } \\
\text { período })\end{array}$ & $\begin{array}{l}\text { Absoluta } \\
\text { - } \\
\text { Números } \\
\text { inteiros, } \\
\text { de zero } \\
\text { até } \\
\text { infinito. }\end{array}$ & $\begin{array}{l}\text { Quantidade total das demandas de alteração a } \\
\text { implementar no sistema. } \\
\text { Propósito: } \\
\text { Verificar o aumento do tamanho de backlog } \\
\text { pode indicar problemas na capacidade de } \\
\text { entrega das demandas aos solicitantes. } \\
\text { Procedimento de coleta: } \\
\text { 1. Contar número de demandas abertas no } \\
\text { período; } \\
\text { 2. Contar número de demandas satisfeitas no } \\
\text { período. Por satisfeitas, será considerado a } \\
\text { disponibilização da demanda em produção para } \\
\text { o solicitante; } \\
\text { 3. Identificar backlog do período anterior. }\end{array}$ \\
\hline
\end{tabular}


Avaliação Pró-ativa da Deterioração de Sistemas de Informação por meio de Medidas de Gestão

Tabela 3 (cont.). Medidas para monitoração da deterioração de sistemas de informação

\begin{tabular}{|c|c|c|c|c|}
\hline Questão & Medida & Fórmula & Escala & Descrição \\
\hline $\mathrm{Q} 1$ & $\begin{array}{l}\text { M3 - } \\
\text { Tamanh } \\
\text { o de } \\
\text { Esforço } \\
\text { por } \\
\text { Demand } \\
\text { a [2] }\end{array}$ & $\begin{array}{l}\left(\sum\right. \\
\text { Tamanho } \\
\text { demandas } \\
\text { satisfeitas) } \\
\text { / } \\
\text { Quantidad } \\
\text { e de } \\
\text { demandas } \\
\text { satisfeitas. }\end{array}$ & $\begin{array}{l}\text { Razão - } \\
\text { Número } \\
\text { s reais } \\
\text { positivo } \\
\text { s. }\end{array}$ & $\begin{array}{l}\text { Tamanho do esforço médio por demanda } \\
\text { entregue no período, apurado em horas. } \\
\text { Propósito: } \\
\text { Ao verificar o esforço médio das demandas, a } \\
\text { medida pode indicar se as solicitações de } \\
\text { modificação estão cada vez maiores } \\
\text { comprometendo a manutenibilidade do } \\
\text { sistema. } \\
\text { Procedimento de coleta: } \\
\text { 1. Identificar as demandas satisfeitas no } \\
\text { período; } \\
\text { 2. Calcular somatório do tamanho das } \\
\text { demandas satisfeitas no período em horas. } \\
\text { Caso este tamanho tenha sido estimado com } \\
\text { alguma ferramenta de dimensionamento de } \\
\text { sistemas, como pontos de função, este valor } \\
\text { deve ser convertido em horas; } \\
\text { 3. Calcular a quantidade total de demandas } \\
\text { satisfeitas no período. }\end{array}$ \\
\hline $\begin{array}{l}\text { Q1 } \\
\text { Q3 }\end{array}$ & $\begin{array}{l}\text { M4 - } \\
\text { Produtiv } \\
\text { idade } \\
\text { Invertid } \\
\text { a } \\
\text { (Adapta } \\
\text { da de } \\
\text { [4]) }\end{array}$ & $\begin{array}{l}\text { (Número } \\
\text { demandas } \\
\text { abertas/ } \\
\text { Número } \\
\text { de } \\
\text { demandas } \\
\text { satisfeitas) } \\
* 100\end{array}$ & $\begin{array}{l}\text { Razão - } \\
\text { Número } \\
\text { s reais } \\
\text { positivo } \\
\text { s. }\end{array}$ & $\begin{array}{l}\text { Percentual de demandas ainda não atendidas } \\
\text { Propósito: } \\
\text { Verificar se o nível de produtividade está } \\
\text { sendo comprometido, prejudicando a } \\
\text { manutenibilidade. Esta métrica teve sua forma } \\
\text { original de cálculo invertida permitindo que } \\
\text { sua interpretação seja semelhante às outras } \\
\text { medidas. } \\
\text { Procedimento de coleta: } \\
\text { 1. Coletar a quantidade total de demandas } \\
\text { criadas no período; } \\
\text { 2. Coletar a quantidade de demandas } \\
\text { satisfeitas no período. }\end{array}$ \\
\hline Q1 & $\begin{array}{l}\text { M5 - } \\
\text { Novas } \\
\text { demand } \\
\text { as }\end{array}$ & $\begin{array}{l}\text { Número } \\
\text { de novas } \\
\text { demandas }\end{array}$ & $\begin{array}{l}\text { Absoluta } \\
- \\
\text { Números } \\
\text { inteiros } \\
\text { positivos. }\end{array}$ & $\begin{array}{l}\text { Número de novas demandas solicitadas no } \\
\text { período } \\
\text { Propósito: } \\
\text { Verificar impacto da carga de trabalho no } \\
\text { resultado de outras medidas. } \\
\text { Procedimento de coleta: } \\
\text { 1. Coletar o número de demandas criadas no } \\
\quad \text { período. }\end{array}$ \\
\hline
\end{tabular}


Tabela 3 (cont.). Medidas para monitoração da deterioração de sistemas de informação

\begin{tabular}{|c|c|c|c|c|}
\hline Questão & Medida & Formula & Escala & Descrição \\
\hline $\mathrm{Q} 1$ & $\begin{array}{l}\text { M6 - } \\
\text { Eficiênc } \\
\text { ia do } \\
\text { Ciclo de } \\
\text { Correçõ } \\
\text { es } \\
\text { (Adapta } \\
\text { da de } \\
{[9] \text { ) }}\end{array}$ & $\begin{array}{l}\sum \text { Tempo } \\
\text { de } \\
\text { Correções } \\
\text { de } \\
\text { Falhas/Nú } \\
\text { mero de } \\
\text { Falhas }\end{array}$ & $\begin{array}{l}\text { Razão - } \\
\text { Número } \\
\text { s reais } \\
\text { positivo } \\
\text { s. }\end{array}$ & $\begin{array}{l}\text { Tempo médio para a resolução das falhas } \\
\text { encontradas na operação do sistema. } \\
\text { Propósito: } \\
\text { Verificar se o tempo necessário para a } \\
\text { correção de falhas no sistema está sendo } \\
\text { comprometido, indicando problemas com a } \\
\text { manutenibilidade do código. } \\
\text { Procedimento de coleta: } \\
\text { 1. Coletar a quantidade de falhas do sistema; } \\
\text { 2. Coletar a quantidade de horas necessárias } \\
\quad \text { para corrigir as falhas identificadas. }\end{array}$ \\
\hline Q2 & $\begin{array}{l}\text { M7 - } \\
\text { Custo } \\
\text { médio } \\
\text { por } \\
\text { demand } \\
\text { a }\end{array}$ & $\begin{array}{l}\text { E Custos } \\
\text { das } \\
\text { demandas } \\
\text { satisfeitas } \\
\text { / Qtde } \\
\text { Total de } \\
\text { Demandas }\end{array}$ & $\begin{array}{l}\text { Razão - } \\
\text { Número } \\
\text { s reais } \\
\text { positivo } \\
\text { s. }\end{array}$ & $\begin{array}{l}\text { Média do custo das demandas satisfeitas no } \\
\text { período. } \\
\text { Propósito: } \\
\text { Verificar se existe uma indicação de um } \\
\text { maior comprometimento do orçamento da } \\
\text { empresa com a manutenção evolutiva do } \\
\text { sistema. }\end{array}$ \\
\hline
\end{tabular}

\section{Procedimento de coleta:}

1. Coletar o custo das demandas satisfeitas;

2. Coletar a quantidade total de demandas satisfeitas.

\begin{tabular}{|c|c|c|c|c|}
\hline Q2 & $\begin{array}{l}\text { M8 - } \\
\text { Custo de } \\
\text { Operação } \\
{[6]}\end{array}$ & $\begin{array}{l}\sum \text { custos de } \\
\text { Operação } \\
\text { do sistema }\end{array}$ & $\begin{array}{l}\text { Razão - } \\
\text { Números } \\
\text { reais } \\
\text { positivos. }\end{array}$ & $\begin{array}{l}\text { Total dos custos de manutenção do sistema: } \\
\text { custos com licenças de software e custos de } \\
\text { suporte e manutenção do sistema. } \\
\text { Propósito: } \\
\text { Verificar se existe uma indicação de maior } \\
\text { comprometimento do orçamento da empresa } \\
\text { com a operação do sistema. } \\
\text { Procedimento de coleta: } \\
\text { 1. Coletar custos com licenças do software; } \\
\text { 2. Coletar os custos com suporte e } \\
\quad \text { manutenção do sistema. }\end{array}$ \\
\hline
\end{tabular}


Tabela 3 (cont.). Medidas para monitoração da deterioração de sistemas de informação

\begin{tabular}{|c|c|c|c|c|}
\hline Questão & Medida & Formula & Escala & Descrição \\
\hline Q2 & $\begin{array}{l}\text { M9 - } \\
\text { Particip } \\
\text { ação no } \\
\text { Orçame } \\
\text { nto }\end{array}$ & $\begin{array}{l}\text { Custo } \\
\text { Operação } \\
\text { Sistema/ } \\
\text { Custo de TI } \\
\text { da } \\
\text { Organização }\end{array}$ & $\begin{array}{l}\text { Razão - } \\
\text { Números } \\
\text { reais } \\
\text { positivos. }\end{array}$ & $\begin{array}{l}\text { Total dos custos de operação do sistema } \\
\text { dividido pelos custos totais de TI da } \\
\text { organização. } \\
\text { Propósito: } \\
\text { Verificar se existe uma indicação de maior } \\
\text { comprometimento do orçamento da empresa } \\
\text { com a operação do sistema. } \\
\text { Procedimento de coleta: } \\
\text { 1. Calcular o custo de operação do sistema, } \\
\quad \text { conforme especificado para a medida M8; } \\
\text { 2. Coletar o orçamento total de TI da } \\
\quad \text { organização. }\end{array}$ \\
\hline Q3 & $\begin{array}{l}\text { M10 - } \\
\text { Tempo } \\
\text { Médio } \\
\text { entre } \\
\text { Falhas } \\
\text { Invertid } \\
\text { o } \\
\text { (Adapta } \\
\text { da de } \\
{[4] \text { ) }}\end{array}$ & $\begin{array}{l}\text { Quantidade } \\
\text { de Falhas/ } \\
\text { Total de } \\
\text { horas de } \\
\text { Operação }\end{array}$ & $\begin{array}{l}\text { Razão - } \\
\text { Números } \\
\text { reais } \\
\text { positivos. }\end{array}$ & $\begin{array}{l}\text { Número de falhas por hora de operação } \\
\text { Propósito: } \\
\text { A quantidade de falhas por hora de operação } \\
\text { do sistema pode indicar a satisfação dos } \\
\text { usuários com relação à confiabilidade do } \\
\text { sistema. Esta métrica teve sua forma original } \\
\text { de cálculo invertida permitindo que sua } \\
\text { interpretação seja semelhante às outras } \\
\text { medidas. } \\
\text { Procedimento de coleta: } \\
\text { 1. Coletar a quantidade de falhas registradas } \\
\quad \text { no período; } \\
\text { 2. Coletar a quantidade total de horas de } \\
\quad \text { operação do sistema. }\end{array}$ \\
\hline Q3 & $\begin{array}{l}\text { M11 - } \\
\text { Tempo } \\
\text { de } \\
\text { respost } \\
\text { a }\end{array}$ & $\begin{array}{l}\text { Quantidade } \\
\text { de segundos } \\
\text { para } \\
\text { conclusão } \\
\text { das } \\
\text { funcionalida } \\
\text { des do } \\
\text { sistema }\end{array}$ & $\begin{array}{l}\text { Absoluta } \\
- \\
\text { Números } \\
\text { inteiros } \\
\text { positivos. }\end{array}$ & $\begin{array}{l}\text { Tempo necessário para que as principais } \\
\text { funcionalidades do sistema sejam executadas } \\
\text { com sucesso. } \\
\text { Propósito: } \\
\text { Verificar a evolução histórica do tempo de } \\
\text { resposta das principais funcionalidades do } \\
\text { sistema pode indicar a deterioração da } \\
\text { satisfação dos usuários, pois reflete a } \\
\text { dificuldade em utilizar o sistema em operação. } \\
\text { Procedimento de coleta: } \\
\text { 1. Selecionar as principais funcionalidades do } \\
\text { sistema } \\
\text { 2. Monitorar o tempo necessário para a } \\
\text { conclusão de cada uma das funcionalidades } \\
\text { apuradas. }\end{array}$ \\
\hline
\end{tabular}


Tabela 3 (cont.). Medidas para monitoração da deterioração de sistemas de informação

\begin{tabular}{lllll}
\hline Questão & Medida & Formula & Escala & Descrição \\
\hline Q3 & M12 - & \% Utilização & Razão - & Percentual de utilização do processador pelas \\
& Nível & de CPU & Números & funcionalidades do sistema. \\
de & (Unidade & reais & Propósito: \\
utiliza & Central de & positivos. & Alterações no nível de utilização da CPU são \\
& ção do & Processamen & & fatores de variação sobre outras medidas da \\
Hardw & to ou & & abordagem, como tempo de resposta do \\
are & Processador) & & sistema.
\end{tabular}

\section{Procedimento de coleta:}

1. Apurar o valor médio trimestral do \% utilização de CPU pelos processos do sistema.

\section{Aplicação Prática}

Esta seção descreve uma das aplicações do modelo proposto em um sistema de uma empresa de grande porte nacional. As medidas foram coletadas utilizando como base os sistemas que armazenam: os registros relativos à ocorrência de falhas, os requisitos de manutenções evolutivas das aplicações e os valores financeiros necessários para manter os sistemas da organização. No entanto, os dados resultantes da monitoração do ambiente produtivo não foram disponibilizados pela organização. Com isso, as análises das medidas M11 (Tempo de resposta) e M12 (Nível de utilização de hardware) não serão ilustradas.

Para as demais medidas (M1 - Volume de Falhas, M2 - Tamanho de Backlog, M3 Tamanho de Esforço por Demanda, M4 - Produtividade Invertida, M5 - Novas demandas, M6 - Eficiência do Ciclo de Correções, M7 - Custo médio por demanda, M8 - Custo de Operação, M9 - Participação no Orçamento e M10 - Tempo Médio entre Falhas Invertido) definiu-se o período histórico de coleta das informações necessárias para o cálculo de cada medida (ver Tabela 3) de abril/2006 até março/2008. Estes dados foram coletados manualmente e armazenados em uma planilha eletrônica, utilizada como ferramenta de apoio à abordagem. Com isso, têm-se oito períodos para as medidas com periodicidade de apuração trimestral, e dois períodos para as medidas anuais.

O sistema avaliado é um dos principais elementos da cadeia de valor da organização, possui aproximadamente 800 usuários e está em operação há 9 anos. Seus processos são executados sob o regime de operação $24 \times 7$, ou seja, praticamente ininterruptos, com uma grande quantidade de informações sendo processadas através de serviços batch e com uma expressiva quantidade de integrações com os outros sistemas da organização.

Este sistema teve seus componentes desenvolvidos parte na linguagem de programação C e parte em COBOL. Seu modelo computacional possui tanto características de aplicações cliente-servidor, com os componentes desenvolvidos em $\mathrm{C}$, quanto mainframe, com seus componentes desenvolvidos em COBOL. 
Avaliação Pró-ativa da Deterioração de Sistemas de Informação por meio de Medidas de Gestão

A Figura 1 apresenta o painel consolidado de medidas para esse sistema. As Figuras 2, 3 e 4 apresentam os painéis detalhados para cada uma das questões de avaliação.

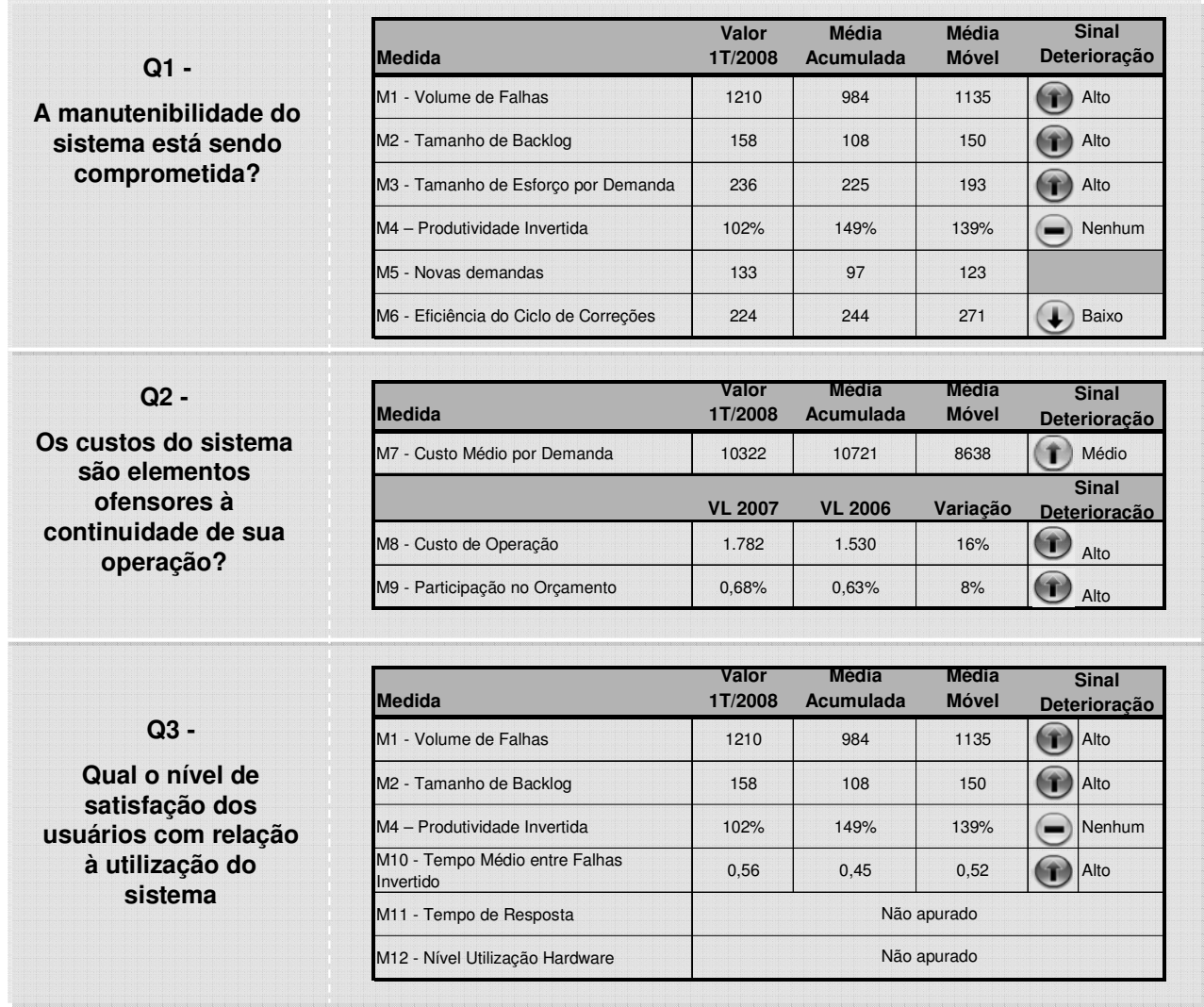

Figura 1. Painel Consolidado de medidas - sistema S1

De acordo com o painel consolidado (Figura 1), praticamente todas as medidas apuradas no 1T/2008 apresentam algum sinal de deterioração, de acordo com os critérios de decisão definidos. Analisando as medidas que visam responder à questão Q1, M1 indica que a quantidade total de falhas apuradas no trimestre foi 1.210. Este número é maior que a média acumulada de todos os períodos (984) e também superior quando comparada à média móvel dos últimos 4 trimestres (1.135). Desta forma, considerando os critérios de decisão definidos, M1 apresenta um alto sinal de deterioração no trimestre apurado. A análise histórica do painel detalhado (Figura 2) ratifica o sinal de deterioração mostrando que nos últimos cinco trimestres, o volume de falhas do sistema apresentou considerável crescimento.

O tamanho do backlog (M2) no 1T/2008 é de 158 demandas. Isso significa que, ao final do trimestre, 158 demandas por manutenção evolutiva foram solicitadas e ainda não 
concluídas. Este número é maior que a média acumulada de todos os períodos (108) e também superior quando comparada à média móvel dos últimos 4 trimestres (150). Assim como M1, M2 também apresenta um alto sinal de deterioração. Entretanto, o tamanho do backlog é impactado diretamente pelo número de novas demandas (M5), que também apresenta um crescimento da carga de trabalho submetida ao sistema S1. No 1T/2008, foram 133 novas demandas, número superior às médias histórica acumulada (97) e recente (103). O painel detalhado (Figura 2) mostra o alto sinal de deterioração desde o período inicial da monitoração.

M3 representa o tamanho do esforço médio para as demandas, ou seja, a quantidade de horas necessárias para a implementação dos novos requisitos solicitados, que no 1T/2008 foi de 236 horas. Este número é maior que a média acumulada de todos os períodos (225h) e também superior quando comparada à média móvel dos últimos 4 trimestres (193h). M3 também apresenta um alto sinal de deterioração no 1T/2008. Historicamente (Figura 2), também apresenta um histórico de alto sinal de deterioração, com exceções registradas no 2T/2007 e 3T/2007. Entretanto, fazendo uma relação dos valores históricos apresentados por estas duas medidas, M2 e M3, percebe-se que o resultado positivo apresentado por M3 no 2T/2007 reflete que somente as demandas de menor tamanho conseguiram ser entregues, ocasionando o aumento do backlog neste período.

M4 indica a produtividade invertida, que é relação entre a quantidade de demandas criadas e entregues, e que no 1T/2008 foi de $102 \%$, ou seja, o número de demandas criadas foi $2 \%$ superior ao número de demandas entregues. Este número é menor que a média acumulada de todos os períodos $(149 \%)$ e também inferior quando analisada frente à média móvel dos últimos quatro trimestres (139\%). Desta forma, M4 não indica nenhum sinal de deterioração no 1T/2008.

O desempenho de M4 é ainda mais significativo quando analisada em conjunto com o volume de Novas Demandas (M5). Ou seja, mesmo com o aumento ocorrido no número de demandas criadas, a produtividade no trimestre não apresentou sinal de deterioração. Entretanto, valores acima de $100 \%$ indicam que mais demandas são criadas do que entregues no período.

Finalmente, M6 representa a quantidade média de horas necessárias para corrigir as falhas ocorridas no sistema durante a sua operação, que no 1T/2008 foi de $224 \mathrm{~h}$. Este número é menor que a média acumulada de todos os períodos (244h) e também inferior quando comparada à média móvel dos últimos quatro trimestres (271). Desta forma, M6 apresenta um baixo sinal de deterioração, pois, mesmo com os valores apurados no trimestre apresentando reduções, a média recente dos últimos quatro trimestres é superior à média histórica acumulada. A análise histórica da eficiência do ciclo de correções (M6), demonstra uma recuperação pelo terceiro trimestre seguido, confirmando a ausência de sinais de deterioração apontados pelo resultado no 1T/2008 (ver Figura 2 - M6). 


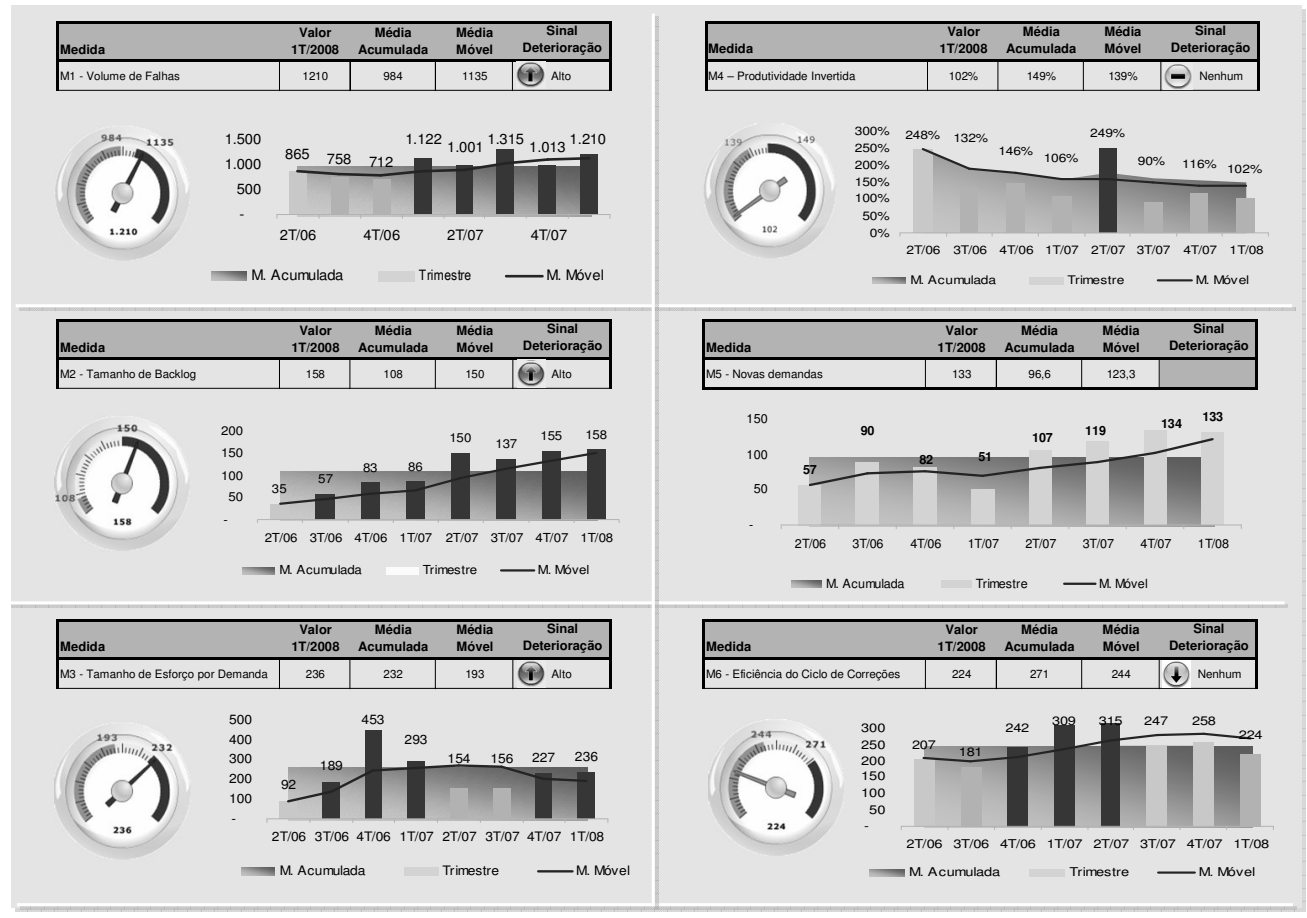

Figura 2. Painel detalhado de medidas para o sistema avaliado - Questão 1

Em síntese, a respeito das medidas relacionadas com a Q1, S1 apresenta, no 1T/2008, um volume crescente de falhas, entretanto, o tempo necessário para a correção destas falhas apresentou resultados melhores que os dados históricos. O aumento no número de novas demandas (M5) pode estar afetando negativamente o desempenho da medida M2, tamanho do backlog, que apresentou crescimento. Mesmo apresentando valores de produtividade melhores que os dados históricos, no 1T/2008, um número maior de demandas foi criado do que entregue fato que pode estar sendo influenciado pelo aumento na quantidade de horas necessárias para a implantação de novas demandas evolutivas.

Quanto à questão Q2, as medidas apresentaram resultados maiores que os dados históricos no 1T/2008. O custo médio por demanda, M7, apresentou uma discreta redução quando comparada com sua média acumulada, entretanto, o desempenho quando comparado com a média móvel é significativamente pior. Desta forma, apresenta um médio sinal de deterioração. O custo de manutenção (M8) em 2007 foi de $\mathrm{R} \$ 1,7$ milhões, frente a R \$ 1,5 milhões de 2006. Os dados históricos (Figura 3) demonstram um crescimento destes custos, fato que é ratificado pelo aumento na participação do custo de manutenção de $S 1$, medida M9, no orçamento da organização de TI. Esta participação aumentou de 0,63\% em 2006 para $0,68 \%$ em 2007. Ambas as medidas apresentam um alto sinal de deterioração. 


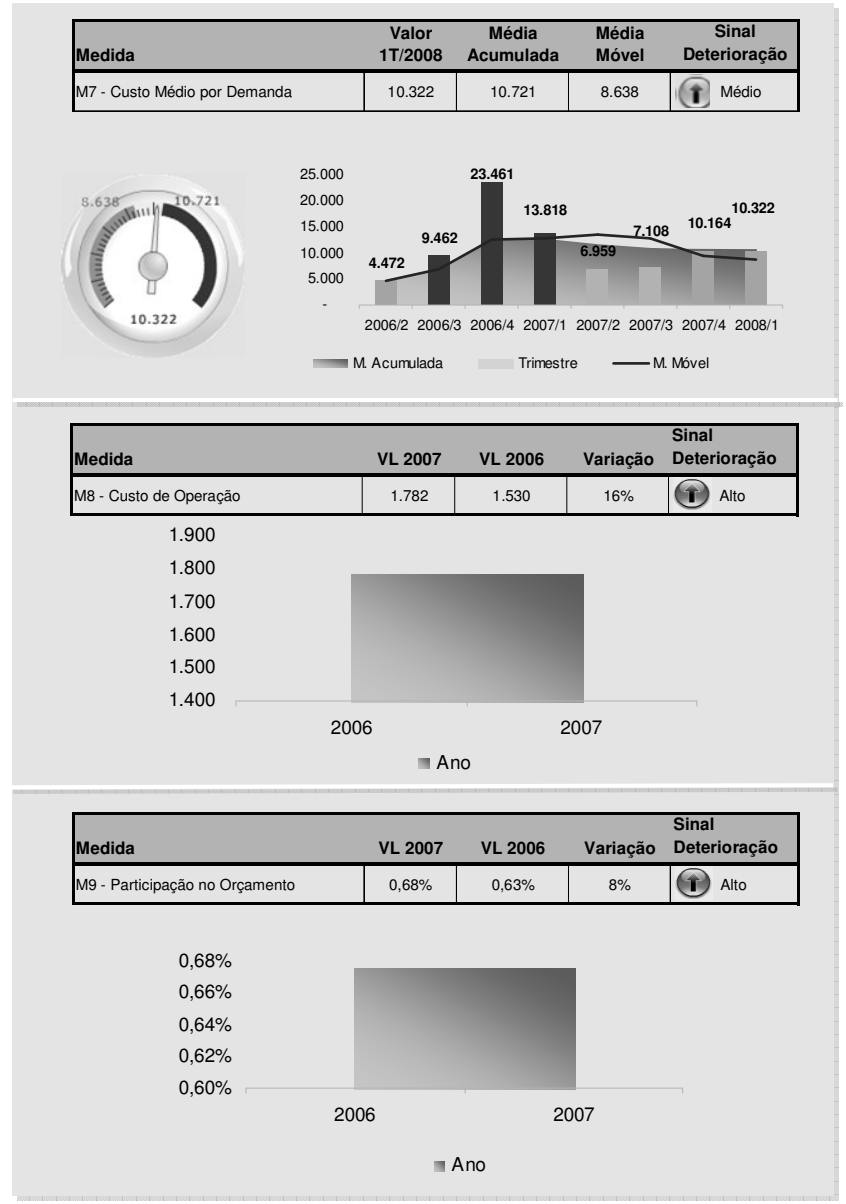

Figura 3. Painel detalhado de medidas para o sistema avaliado - Questão 2

De forma geral, no trimestre apurado, a análise da questão Q2 para o sistema S1 apresenta um aumento nos custos de evolução do sistema, nos custos de operação e uma participação maior no orçamento da organização de TI.

O nível de satisfação dos usuários, representada pela Q3, reúne as medidas $\mathrm{M} 1, \mathrm{M} 2$, M4, M10, M11 e M12. Como visto anteriormente, M1 e M2 apresentaram alto sinal de deterioração no 1T/2008. Nenhum sinal foi identificado em M4, entretanto valores superiores que $100 \%$ indicam que mais demandas são criadas do que entregues no período. M10 indica que considerando o tempo total de operação e a quantidade de falhas ocorridas no 1T/2008, na média, ocorrem 0,56 falhas a cada hora de operação do sistema. Este número é maior que a média acumulada de todos os períodos $(0,45)$ e também superior quando comparada à média móvel dos últimos quatro trimestres $(0,52)$. De acordo com os critérios de decisão 
definidos, M10 apresenta alto sinal de deterioração no trimestre apurado, sendo confirmado pelos dados históricos (Figura 3) que mostra o aumento sucessivo de falhas por hora de operação do sistema ocorrido nos últimos trimestres.

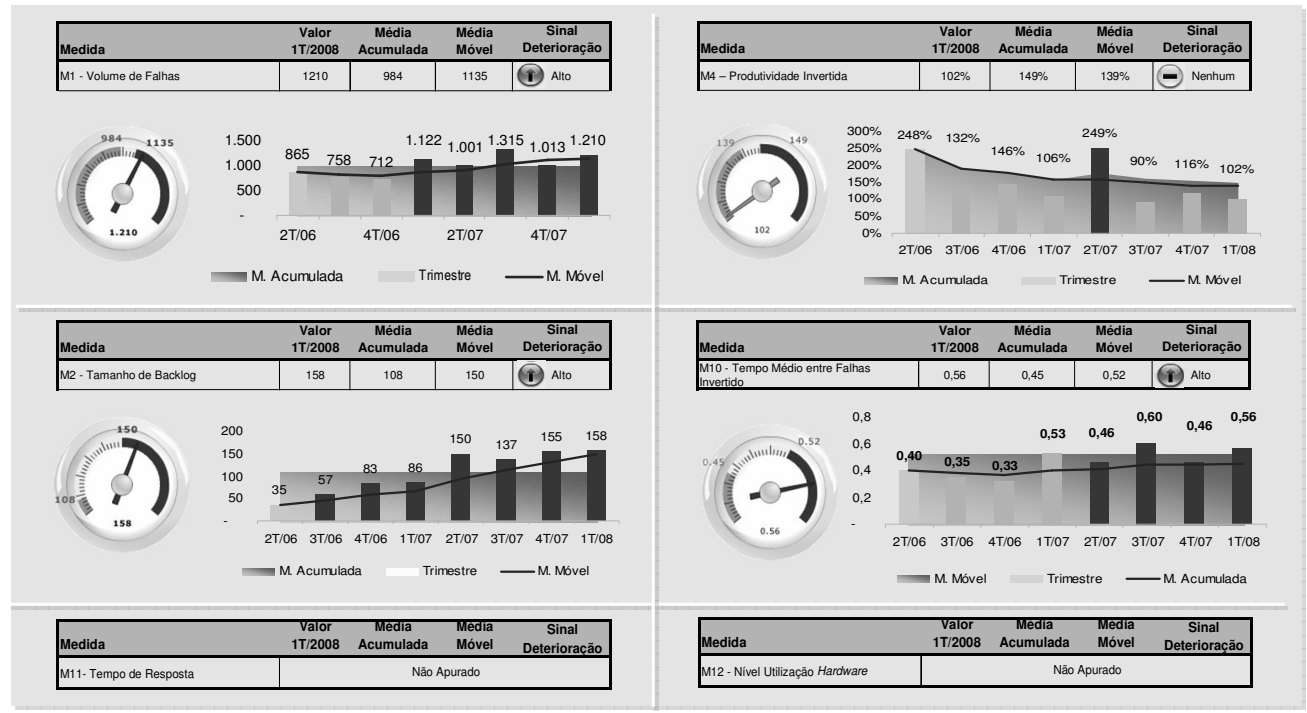

Figura 4. Painel detalhado de medidas para o sistema avaliado - Questão 3

Em resumo, de acordo com as medidas definidas para a questão Q3 e apuradas no 1T/2008, na visão dos usuários, mais falhas ocorreram no trimestre, o tamanho do backlog de demandas por evolução do sistema está aumentando e mais falhas ocorrem por hora de operação do sistema.

Dessa forma, pode-se concluir que esse sistema apresenta sinais de deterioração, fato confirmado pelos seus respectivos gestores que, após o 1T/2008, iniciaram uma investigação mais detalhada visando avaliar a substituição de alguns de seus módulos. Analisando os painéis consolidados e detalhados é possível identificar que os sinais percebidos de deterioração no 1T/2008 já haviam se manifestado em períodos anteriores. O aumento do volume de falhas e do tempo necessário para sua correção, por exemplo, apresentaram altos sinais de deterioração desde o 1T/2007, sendo confirmados a partir do 2T/2007. Neste mesmo trimestre, com o aumento no número de novas demandas, houve um comprometimento do backlog e da produtividade do sistema. Assim, com uso desta ferramenta os gestores poderiam ter iniciado ações que reforçassem a qualidade das manutenções evolutivas realizadas visando combater o crescente número de erros, realizar manutenções preventivas no seu código com o objetivo de melhorar a manutenibilidade do sistema e por em curso atividades visando a garantia da qualidade da documentação. 


\section{Conclusões}

Com os atuais níveis de utilização de sistemas de informação nas organizações, nos mais diversos objetivos, torna-se necessário a relevância de ações de gestão que visem garantir a "saúde" destes ativos. Como uma destas ações, destaca-se a pró-atividade na detecção da deterioração destes sistemas, da otimização do uso destes recursos e da possibilidade do tratamento de problemas em seus estágios iniciais.

Este trabalho propôs um conjunto de medidas, definidas por meio da abordagem QGM, com o objetivo de monitorar a deterioração de sistemas de informação, na visão de gestores das organizações de TI. Essas medidas foram definidas a partir da literatura sobre métodos de apoio a decisão sobre modernização de sistemas e de entrevistas com gestores. No entanto, ao contrário das propostas desses métodos que buscam já identificar a melhor alternativa de modernização para um sistema, na proposta deste trabalho as medidas foram detalhadamente analisadas e descritas para permitir uma avaliação contínua do sistema e de forma pró-ativa sinalizar sobre sua deterioração o mais cedo possível, possibilitando aos gestores agir antecipadamente. Para isso foi decidido utilizar medidas que permitissem evolução histórica, fossem de coleta simples e usando avaliação objetiva, diferentemente da proposta de Harris e Mcroy [8], único trabalho encontrado que apresenta algum foco na próatividade, que utiliza a avaliação subjetiva para os indicadores propostos. Além da descrição das medidas, duas importantes contribuições deste trabalho, são a definição de critérios de decisão que qualificam os valores apurados para as medidas de monitoração da deterioração dos sistemas e o desenvolvimento de instrumentos para a apresentação, aos gestores, da monitoração da deterioração dos sistemas.

Essa abordagem foi aplicada em diferentes sistemas de uma organização. Outras aplicações em diferentes domínios e instituições devem ser realizadas para melhor avaliar os resultados dessa abordagem. No entanto, a aplicação inicial indica que, além da viabilidade de coleta dos dados, este conjunto de medidas se constitui em um instrumento de gestão no apoio à identificação precoce de necessidade de ações que visam minimizar a deterioração de sistemas de informação, uma vez que permitem evidenciar problemas na capacidade em manter e evoluir um sistema, com os custos envolvidos na sua utilização e com a satisfação de seus usuários. Além disso, a abordagem mostrou-se fácil de aplicar, pois a descrição dos procedimentos de coleta das medidas é simples e os instrumentos de apresentação dos resultados utilizam apenas planilhas eletrônicas. Dessa forma, para novas aplicações é necessário apenas o entendimento das medidas e de como realizar sua coleta na instituição desejada.

Além da aplicação da abordagem em novos contextos, pretende-se como trabalhos futuros refinar os critérios de decisão utilizados, avaliar a viabilidade de inclusão de medidas que reflitam informações relacionadas diretamente com o processo automatizado pelo sistema e analisar diferentes decisões que podem ser tomadas pelos gestores para os diferentes graus de deterioração identificados. 


\section{Referências}

1. Lintinen, H.; Koskinen, J.; Ahonen, J.; Sivula, H.; Tilus, T. "Software Modernizations - A Qualitative Analysis of Industrial Decision Making”. ELTISproject, Information Technology Research Institute (TITU), Univ. of Jyväskylä. Technical report, 2004.

2. Sneed, H. "Planning the reengineering of legacy systems". IEEE Software 12 (1), p. 24-34, 1995.

3. Ransom, J., Sommerville, I. e Warren, I. "A method for assessing legacy systems for evolution". Proc. 2nd Euromicro Conference on Software Maintenance and Reengineering, 1998.

4. Visaggio, G. "Value-based decision model for renewal processes in software maintenance". Annals of Software Engineering 9, p. 215-233, 2000.

5. De Lucia, A., Fasolino, A.R. e Pompelle, E. "A Decisional Framework for Legacy System Management”, p. 642-651, 2001.

6. Aversano, L., Esposito, R., Mallardo, T. e Tottorella, M. "Supporting Decisions on the Adoption of Re-engineering Technologies". Proceedings of the Eighth European Conference on Software Maintenance and Reengineeringm, 2004.

7. Koskinen, J., Ahonen, J., Sivula, H., Tilus, T, Lintinen, H. e Kankaanpaa, I. "Software Modernization Decision Criteria: An Empirical Study". Proceedings of the Ninth European Conference on Software Maintenance and Reengineering, 2005.

8. Harris, K., Mcroy, L. "Use a Health Check to Determine Your Application's 'Fitness for Duty"'. Gartner Research. 2006.

9. ISO/IEC TR 9126-2 "Software engineering - Product quality - Part2 :External metrics", 2002.

10. Bennett, K., Ramage, M., Munro, M. "Decision Model for Legacy Systems". IEE Proceedings Software, vol. 146, n 3, p. 153-159, 1999.

11. Lehman, M.; Ramil, J. F.; Wernick, P. ; Perry, D.; Turski, W. "Metrics and Laws of Software Evolution - The Nineties View", Fourth International Software Metrics Symposium 1997.

12. Jermakovics, A.; Scotto, M.; Succi, G. "Visual Identification of Software Evolution Patterns”. IWPSE'07, p; 27-30, Dubrovnik, Croatia, 2007.

13. Gurp, J.; Bosch, J. "Design erosion: problems and causes. Journal of Systems and Software", v.61 n.2, p.105-119, 2002.

14. Parnas, D. "Software Aging". Proc. 16th Int'l Conf. Software Eng., pp. 279-287, 1994. 
15. Eick S.; Graves, T.; Karr, A; Marron, J.; Mockus, A. "Does Code Decay? Assessing the Evidence from Change Management Data". IEEE Transactions on Software Engineering, v.27 n.1, p.1-12, 2001.

16. Cimitile, A.; Fasolino, A.; Lanubile, F. "Legacy Systems Assessment to Support Decision Making”, 2001. Disponível em < http://citeseer.ist.psu.edu/491707.html> Acesso em: 29 Mar. 2008.

17. Kajko-Mattsson, M. "Preventive Maintenance! Do We Know What It Is?". Proceedings, International Conference on Software Maintenance, 2000.

18. Basili, V. R., Caldiera, G. and Rombach, H. D. "The Goal Question Metric Paradigm”. Encyclopedia of Software Engineering, vol. 1, p. 528-32, 1994.

19. Soligen, R. and Berghout, E. "The Goal Question Metric Method - A Practical Guide for Quality Improvement of Software Development", Great Britain: Cambridge, McGraw-Hill, 1999.

20. Kithchenham, B.; Walker, J. A Quantitative Approach to Monitorig Software Development. Software Engineering Journal, p. 2-13, 1989

21. Fenton, N.; Neil, M. Software metrics: roadmap. Proceedings of ht conference on The Future of Software Enginnering, pp.357-370, Limerick, Ireland, 2000.

22. ISO/IEC FDIS 15939. Software engineering - Software measurement process. 2002.

23. Pickard, M. Analysis of software metrics. In: Proc. Centre for Software Reliability Conf. on Measurement for Softw. Control and Assurance (Eds) Kitchenham, B. and Littlewood, B. Bristol U.K. Elsevier p. 155-180. 1989

24. Visaggio, G. "Assessment of a Renewal Process Experimented in the Field," The Journal of Systems and Software 45, 1, p. 3-17, 1999. 